The Astrophysical Journal, 450:L59-L62, 1995 September 10

(c) 1995. The American Astronomical Society. All rights reserved. Printed in U.S.A.

\title{
BALMER DISCONTINUITY TEMPERATURES IN THE ORION NEBULA
}

\author{
X.-W. LiU AND M. J. BARLOW \\ Department of Physics and Astronomy, University College London, Gower Street, London, WC1E 6BT, UK \\ I. J. DANZIGER \\ European Southern Observatory, Karl-Schwarzschild Str., D-85748 Garching bei München, Germany \\ AND \\ P. J. STOREY \\ Department of Physics and Astronomy, University College London, Gower Street, London WC1E 6BT, UK \\ Received 1995 May 22; accepted 1995 July 6
}

\begin{abstract}
We have mapped the Balmer discontinuity temperature $T_{e}(\mathrm{BJ})$ along two long-slit positions in the Orion Nebula, using high-quality spectra obtained with the ESO $1.52 \mathrm{~m}$ telescope and with the $3.9 \mathrm{~m}$ AAT. One slit was oriented north-south and positioned 40" west of $\theta^{1} \mathrm{C}$ Ori. The second slit was oriented east-west, with its eastern end $10^{\prime \prime}$ west of $\theta^{1} \mathrm{C}$ Ori, identical to the slit position previously studied by Walter \& Dufour (1994). For the NS slit, both the $1.52 \mathrm{~m}$ and the AAT data yield a constant temperature of about $9000 \mathrm{~K}$, with variations of only a few hundred K over a total slit length of about 3.5. For the EW-slit, our data reveal two distinct regions of nearly constant temperatures of 8900 and $7200 \mathrm{~K}$, respectively, possibly resulting from two separate $\mathrm{H}$ II regions. No evidence is found for the anomalously low temperatures reported by Walter and Dufour for the same region. Subject headings: H II regions — ISM: abundances — ISM: individual (Orion Nebula)
\end{abstract}

\section{INTRODUCTION}

Recent abundance studies of a number of $\mathrm{H}$ II regions and planetary nebulae (PNs) using optical recombination lines (Peimbert, Storey, \& Torres-Peimbert 1993; Liu et al. 1995; Barlow et al., in preparation) suggest that the "standard" method based on forbidden-line analyses (see e.g., Osterbrock 1989) may underestimate the real abundances of heavy elements by a factor of 2-3. Evidence of this effect was first given by Peimbert $(1967,1971)$ who measured electron temperatures for three PNs and the Orion Nebula (M42) using the ratio $I_{c}(\mathrm{BJ}) / I(\mathrm{H} \beta)$ of the nebular Balmer jump to $\mathrm{H} \beta$, and found systematically lower temperatures than derived from the [O III $] \lambda 5007 / \lambda 4363$ ratios for the same objects. He suggested that $\mathrm{H}$ II regions and PNs have significant temperature fluctuations, so that temperatures derived from forbidden line ratios would overestimate the true mean temperature, while abundances derived from these forbidden lines would consequently be underestimated. Liu \& Danziger (1993) measured $T_{e}(\mathrm{BJ})$ and $T_{e}([\mathrm{O} \mathrm{III}])$ for $14 \mathrm{PNs}$ and found that on average $T_{e}(\mathrm{BJ})$ tends to be lower than $T_{e}([\mathrm{O} \mathrm{III}])$ by $\sim 1400 \mathrm{~K}$. Recently, Walter \& Dufour (1994, hereafter WD94) mapped $T_{e}(\mathrm{BJ})$ along a long slit positioned on M42 and found it to decrease from 8400 to $2800 \mathrm{~K}$ between $40^{\prime \prime}$ and $220^{\prime \prime}$ west of $\theta^{1} \mathrm{C}$ Ori. This result, if confirmed, casts serious doubt on the reliability of $I_{c}(\mathrm{BJ}) / I(\mathrm{H} \beta)$ as a thermometer, since it is difficult to understand temperatures as low as $2800 \mathrm{~K}$ in a photoionized nebula such as M42. Given the potential importance of their result, we have mapped $T_{e}(\mathrm{BJ})$ for two regions in $\mathrm{M} 42$, including the area studied by WD94. The results are described here.

We use the ratio of $I_{c}(\mathrm{BJ})$ to the intensity of $\mathrm{H} 11$ at $3770 \AA$, instead of relative to the intensity of $\mathrm{H} \beta$, to determine $T_{e}(\mathrm{BJ})$. Because of the small wavelength difference between the Balmer discontinuity and H11, this approach minimizes possible effects due to systematic errors (e.g., atmospheric differ- ential refraction, atmospheric extinction, and flux calibration). Furthermore it has the great advantage that the wavelength dependence of the reddening correction toward M42, known to be anomalous, becomes largely irrelevant. For example, using the extinction law derived for the Trapezium stars by Cardelli, Clayton, \& Mathis (1989), we find that $I_{c}(\mathrm{BJ}) / I(\mathrm{H} 11)$ changes by only $2.4 \%$ when $c(\mathrm{H} \beta)$, the logarithmic extinction at $\mathrm{H} \beta$, increases from zero to one. For the regions of M42 of interest here, the extinction falls well within this range (Baldwin et al. 1991, hereafter B91). Thus in our case, $I_{c}(\mathrm{BJ}) /$ $I(\mathrm{H} 11) \equiv F_{c}(\mathrm{BJ}) / F(\mathrm{H} 11)$, where we use $I$ and $F$ to denote intrinsic and observed fluxes, respectively. The emissivities as functions of electron temperature for the nebular continuum and the $\mathrm{H}$ I Balmer lines are taken from Brown \& Mathews (1970) and Storey \& Hummer (1995), respectively. To take account of the contribution from a small discontinuity at 3680 $\AA$ produced by recombinations to the $\mathrm{He}$ I $2{ }^{1} \mathrm{P}^{o}$ level, which is inseparable from the Balmer discontinuity, we follow Liu \& Danziger (1993) and define the Balmer discontinuity as $I_{c}(\mathrm{BJ}) \equiv I_{c}(\lambda 3643)-I_{c}(\lambda 3681)$, where $I_{c}(\lambda 3643)$ and $I_{c}(\lambda 3681)$ are the nebular continuum fluxes at 3643 and $3681 \AA$, respectively. A power-law fit to the relationship between $I_{c}(\mathrm{BJ}) /$ $I(\mathrm{H} 11)$ and $T_{e}$ is given by $T_{e}(\mathrm{BJ})=382.5 \times\left[I_{c}(\mathrm{BJ}) /\right.$ $I(\mathrm{H} 11)]^{-1.492} \mathrm{~K}$, with a maximum error of $3.5 \%$ for $T_{e}$ between 4000 and $20,000 \mathrm{~K}$. A better fit can be achieved with a cubic spline function, which is used here. A diagram showing both fits is available on request (contact xwl@star.ucl.ac.uk). We have assumed $\mathrm{He}^{+} / \mathrm{H}^{+}=0.09$ (B91); neglect of the He I discontinuity at $3680 \AA$ would underestimate $T_{e}$ by $200 \mathrm{~K}$.

\section{OBSERVATIONS}

On 1994 December 20-24 M42 was observed using the B\&C Spectrograph of the ESO $1.52 \mathrm{~m}$ telescope. The slit was oriented north-south and positioned through the star JW 423 (Jones \& Walker 1988), 40".8 west and 75."6 north of $\theta^{1}$ C Ori. 

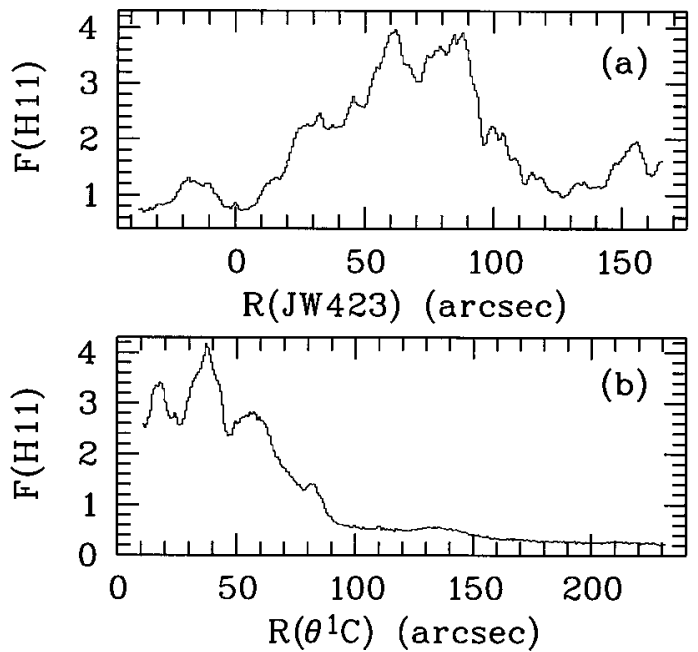

FIG. 1.-F(H11), the observed surface brightness distributions of $\mathrm{H} 11$ (in units of $10^{-14} \mathrm{ergs} \mathrm{cm}^{-2} \mathrm{~s}^{-1} \operatorname{arcsec}^{-2}$ ), deduced from (a) the NS slit, ESO 1.52 $\mathrm{m}$ data; and $(b)$ the EW slit, AAT data. $R(\mathrm{JW} 423)$ and $R\left(\theta^{1} \mathrm{C}\right)$ are the angular distances south of JW 423 and west of $\theta^{1} \mathrm{C}$ Ori, respectively. $R(\mathrm{JW} 423)$ and $R\left(\theta^{1} \mathrm{C}\right)$ are accurate to within 0.1 and $1^{\prime \prime}$, respectively. The NS- and the $\mathrm{EW}$-slits intersect at $R(\mathrm{JW} 423)=75 . " 6$ and $R\left(\theta^{1} \mathrm{C}\right)=40 " 8$.

The detector was a $2048 \times 2048$ pixel Ford CCD and each pixel along the slit projected to 0.815 on the sky. Several gratings were used to provide coverage from 3410 to $8770 \AA$. Spectra of $\theta^{1}$ A, B, C, and D Ori were obtained for two slit widths: $2^{\prime \prime}$ and $6 "$. Here we discuss only spectra covering $\lambda \lambda 3410-4410$ from a single grating setup at a resolution of $1 \AA$ FWHM. The observations at the $3.9 \mathrm{~m}$ AAT were carried out on 1995 February 8-9 using the RGO Spectrograph, covering 3510 to $3911 \AA$ at $0.8 \AA$ FWHM resolution. The detector was a $1024 \times 1024$ pixel Tektronix CCD and each pixel along the slit projected to 0.772 on the sky. Two slit positions were observed, one identical to the ESO $1.52 \mathrm{~m} \mathrm{~N}-\mathrm{S}$ position, and the other oriented east-west with its eastern end starting $10^{\prime \prime}$ west of $\theta^{1} \mathrm{C}$ Ori. This second slit position was identical to that observed by WD94. Again we observed the four Trapezium stars using the same setup with $2^{\prime \prime}$ and $6 "$ slit widths. All the nebular spectra obtained with the ESO $1.52 \mathrm{~m}$ telescope and with the AAT were secured with a 2 " slit width under typical seeing conditions of 1" FWHM. All the spectra were fluxcalibrated by observing the HST standard star HD 49798 (spectral type O6, $V=8.30$; Walsh 1993). HD 49798 has only weak Balmer absorption lines and a very small Balmer jump. In addition it is calibrated every $6 \AA$ in the near-UV and blue regions. All data were reduced using standard techniques for long-slit spectra.

\section{RESULTS}

The derived surface brightness distributions of $\mathrm{H} 11$ along the NS and EW slits are shown in Figure 1. The mean $T_{e}(\mathrm{BJ})$ derived after integrating along each slit are given in Table 1. For comparison, results are listed both with and without the subtraction of the stellar scattered light and sky background contributions (see below). Table 1 shows that the scattered light and sky background have only marginal effects on the derived $T_{e}(\mathrm{BJ})$. All three of our independent data sets give a mean $T_{e}(\mathrm{BJ})$ of $9000 \mathrm{~K}$ within the estimated errors of less than $300 \mathrm{~K}$.

The total observed continuum flux $F_{c}(\lambda)$ is assumed to be
TABLE 1

Mean Balmer Jump Temperatures in THE ORion Nebula ${ }^{\text {a }}$

\begin{tabular}{cccc}
\hline \hline & \multicolumn{2}{c}{ NS SLIT } & EW SLIT \\
\cline { 2 - 3 } PARAMETER & ESO $1.52 \mathrm{~m}$ & AAT & AAT \\
\hline \multicolumn{3}{c}{ Before Scattered Light and Sky Subtraction } \\
$F(\mathrm{H} 11)^{\mathrm{b}} \ldots \ldots \ldots \ldots$ & 232 & 234 & 132 \\
$F_{c}(\mathrm{BJ})^{\mathrm{c}} \ldots \ldots \ldots \ldots$ & 27.8 & 28.0 & 16.6 \\
$T_{e}(\mathrm{BJ})(\mathrm{K}) \ldots \ldots \ldots$ & 8860 & 8810 & 8230 \\
After Scattered & Light and Sky Subtraction \\
$F(\mathrm{H} 11)^{\mathrm{b}} \ldots \ldots \ldots \ldots$ & 237 & 237 & 134 \\
$F_{c}(\mathrm{BJ})^{\mathrm{c}} \ldots \ldots \ldots \ldots$ & 27.7 & 28.4 & 16.1 \\
$T_{e}(\mathrm{BJ})(\mathrm{K}) \ldots \ldots \ldots$ & 9180 & 8840 & 8800 \\
\hline
\end{tabular}

${ }^{\mathrm{a}}$ For the NS slit, the measurements were made on the spectrum integrated from $4^{\prime \prime}$ to $125^{\prime \prime}$ south of JW 423 . For the EW-slit, the spectrum was integrated from $10^{\prime \prime}$ to $190^{\prime \prime}$ west of $\theta^{1}$ C Ori. The power-law fit given in $\S 1$ yields $T_{e}(\mathrm{BJ}) 2.5 \%-3.1 \%$ higher than those listed here, derived from the more accurate cubic spline fit.

${ }^{b}$ In units of $10^{-16} \mathrm{ergs} \mathrm{cm}^{-2} \mathrm{~s}^{-1} \operatorname{arcsec}^{-2}$.

${ }^{c}$ In units of $10^{-16} \mathrm{ergs} \mathrm{cm}^{-2} \mathrm{~s}^{-1} \operatorname{arcsec}^{-2} \AA^{-1}$.

given by $F_{c}(\lambda)=F_{c}($ neb,$\lambda)+F_{c}($ sca, $\lambda)+F_{c}($ sky, $\lambda)$, where the contributions from the sky background $F_{c}$ (sky, $\lambda$ ) and stellar scattered light, $F_{c}$ (sca, $\lambda$ ) were determined as follows. During the ESO $1.52 \mathrm{~m}$ observing run, a total of eight spectra of M42 covering $\lambda \lambda 3410-4410$, each of 20 minutes duration, were obtained. The first three exposures were secured before the moon rose and we assumed that the sky background was negligible for these spectra. The wavelength dependence of the sky spectrum was then derived from the spectra of two planetary nebulae observed later on the same night, and this was scaled to match the difference between the spectra with and without sky contributions. In order to derive the contribution of stellar scattered light, we made the following assumptions: (1) The scattered light is solely due to the four Trapezium stars, with the contribution from each star being proportional to its apparent brightness; (2) The dust scattering efficiency varies as $\lambda^{-1}$ (Costero \& Peimbert 1970). Thus, the spectral distribution of the scattered light is given by $F_{c}$ (sca, $\lambda)=c\left(\lambda / \lambda_{0}\right)^{-1} F_{c}(\operatorname{Tr}, \lambda)$, where $c$ is a constant, $\lambda_{0}$ is a reference wavelength, and $F_{c}(\operatorname{Tr}, \lambda)$ is the mean continuum flux of the Trapezium stars, formed by averaging the spectra of $\theta^{1} \mathrm{~A}, \mathrm{~B}$, C, and D Ori with weights of 15:3:68:14, their brightness ratios in the blue and near-UV wavelength regions. (Eq. [2] and the relation that leads to it in WD94 are incorrect. The positions of the coefficients 3646 and 4580 should be reversed.) The constant $c$ can be derived once the scattered light contribution at any particular wavelength is known. The magnitude of the nebular continuum, $F_{c}$ (neb, $\lambda 3835$ ), can be inferred from the observed intensity, $F(\mathrm{H} 9)$, of the Balmer line at that wavelength, using the value of $T_{e}(\mathrm{BJ})$ derived before any scattered light or sky subtraction. Note that the value of $F_{c}($ neb, $\lambda 3835)$ derived in this way is independent of the reddening correction toward M42. Using $F_{c}(\mathrm{neb})$ and $F_{c}$ (sky) at one wavelength, $F_{c}$ (sca) can be deduced.

The ESO $1.52 \mathrm{~m}$ spectrum of M42, integrated along the NS slit, is shown in Figure $2 a$. The derived scattered light and sky background contributions are shown in Figure $2 b$, while Figure $2 c$ plots the spectrum after the scattered light and sky subtraction. Also shown in Figures $2 a$ and $2 c$ are polynomial fits to the observed continuum levels. The regions blueward and redward of the Balmer discontinuity are fitted separately. On the longer 


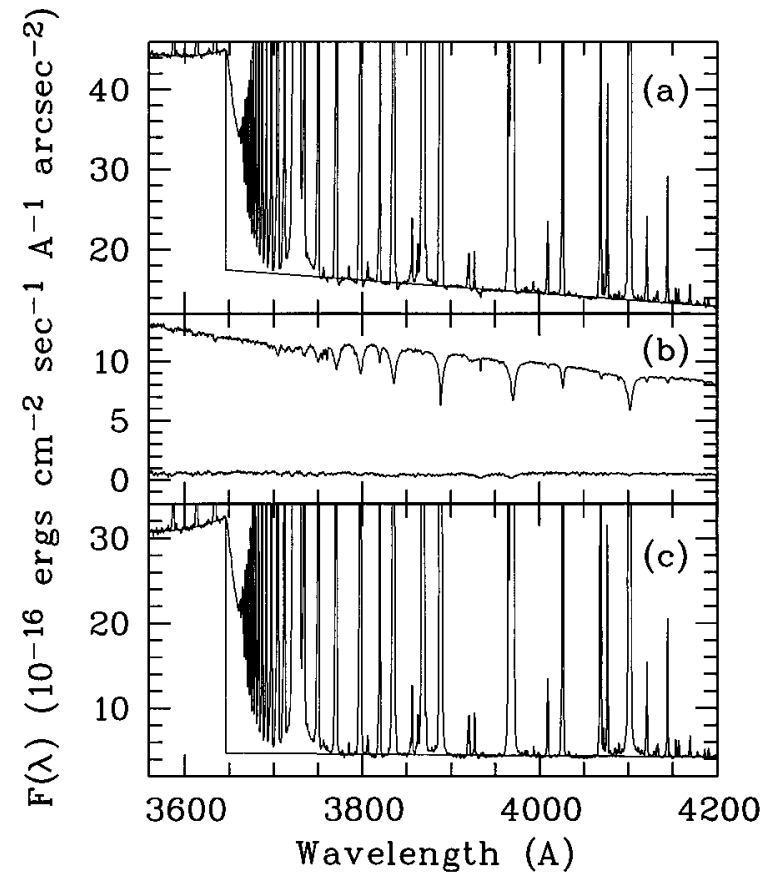

FIG. 2.-ESO $1.52 \mathrm{~m}$ spectra of M42, integrated along the NS-slit from 4" to $125^{\prime \prime}$ south of JW 423. (a) The spectrum before the scattered light and sky background corrections. (b) Upper curve: the estimated stellar scattered light lower curve: the sky background. $(c)$ Spectrum $(a)$ after subtraction of the scattered light and sky contributions shown in $(b)$. Note that the scattered stellar absorption line wings seen in $(a)$ disappear in the corrected spectrum $(c)$. Also shown in $(a)$ and $(c)$ are polynomial fits to the continuum bluewards and redwards of the Balmer jump at $3646 \AA$.

wavelength side of the jump, a straight line is sufficient to fit the continuum level over the whole wavelength range of concern here. However, on the short-wavelength side of the jump, sometimes a second order polynomial is required, especially before the scattered light subtraction. Immediately redwards of the Balmer jump, the spectrum is crowded by high order Balmer lines and, even more seriously, the extended
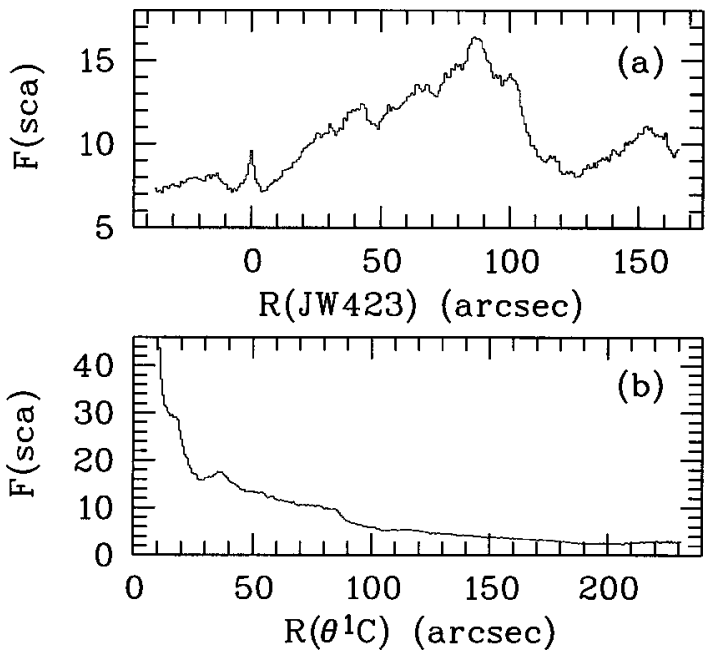

FIG. 4. $-F($ sca $)$, the surface brightness distribution of stellar scattered light at $3810 \AA$ (in units of $10^{-16} \mathrm{ergs} \mathrm{cm}^{-2} \mathrm{~s}^{-1} \operatorname{arcsec}^{-2} \AA^{-1}$ ), along (a) the NS; and (b) the EW slit. The small peak at $R(\mathrm{JW} 423)=0$ in $(a)$ is from JW 423. See also the caption to Fig. 1.

wings of the extremely strong [O II] doublet at $3727 \AA$ distort the nearby continuum. Thus only data points longwards of $3750 \AA$ were used to fit the continuum level longwards of the Balmer jump.

The analyses carried out for the spectra integrated along the slits were applied to each individual spectral scan line along both the NS and EW slits. Examples of spectra at some selected points are shown in Figure 3. The upper three panels show spectra near the peak nebular surface brightness, whereas the lower three are for low surface brightness points (see Fig. 1). The derived surface brightness distributions of scattered light at $3810 \AA$ along the slits are shown in Figure 4. The deduced distributions of $T_{e}(\mathrm{BJ})$ along the NS and EW slits are given in Figure 5. The results deduced both before and after scattered light and sky subtraction are shown. Note that both Table 1 and Fig. 5 show that after correction for the

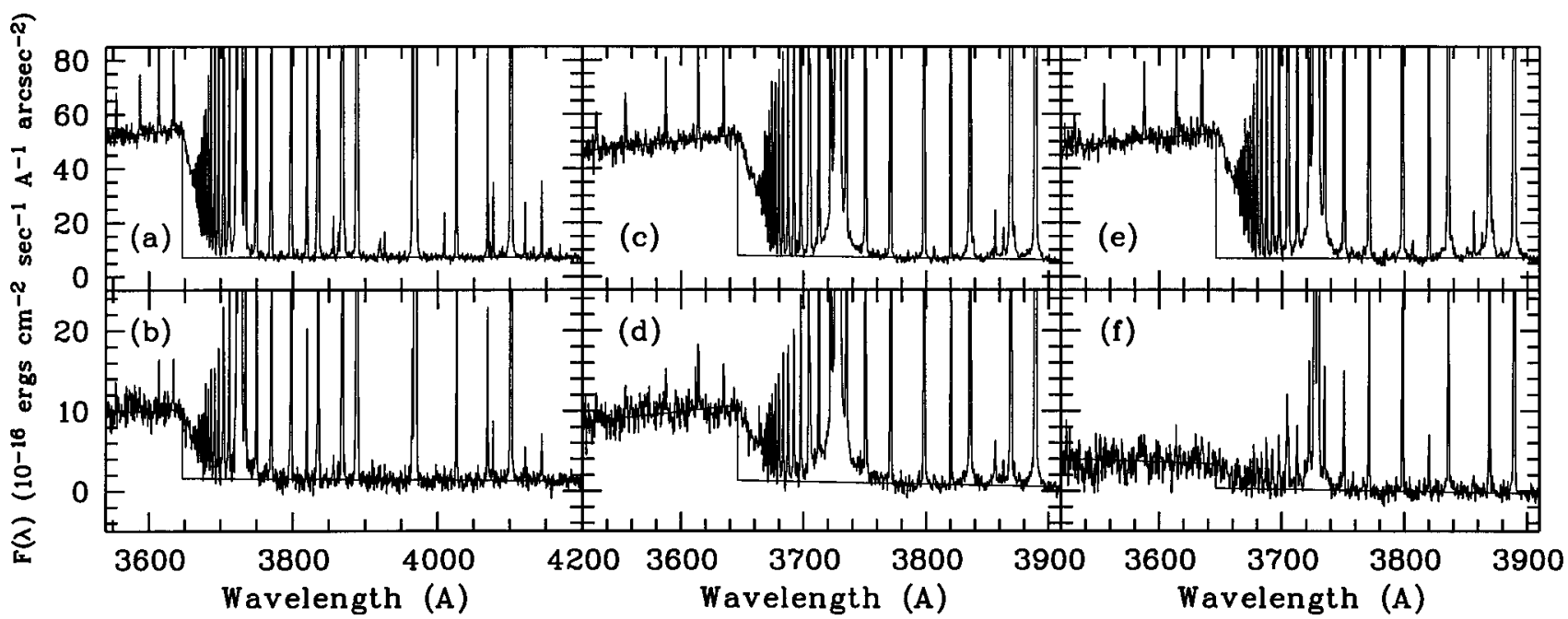

FIG. 3.-Spectra of M42 near the Balmer jump region at some selected points along the NS and the EW slits. Panels $(a)$ and $(b)$ are from the ESO $1.52 \mathrm{~m}$ data while panels $(c-f)$ are from the AAT data. $(a, c) 62^{\prime \prime}$ south of JW 423; $(b, d) 5^{\prime \prime}$ south of JW 423; $(e) 37^{\prime \prime}$ west of $\theta^{1}$ C Ori; $(f) 220^{\prime \prime}$ west of $\theta^{1}$ C Ori. Each spectrum represents one pixel along the slit on the detector, sampling $0.815 \times 2^{\prime \prime}$ and $0.772 \times 2^{\prime \prime}$, respectively, for the ESO $1.52 \mathrm{~m}$ data and the AAT data. Also shown are polynomial fits to the nebular continuum bluewards and redwards of the Balmer jump. The stellar scattered light and sky background contributions have been subtracted. 


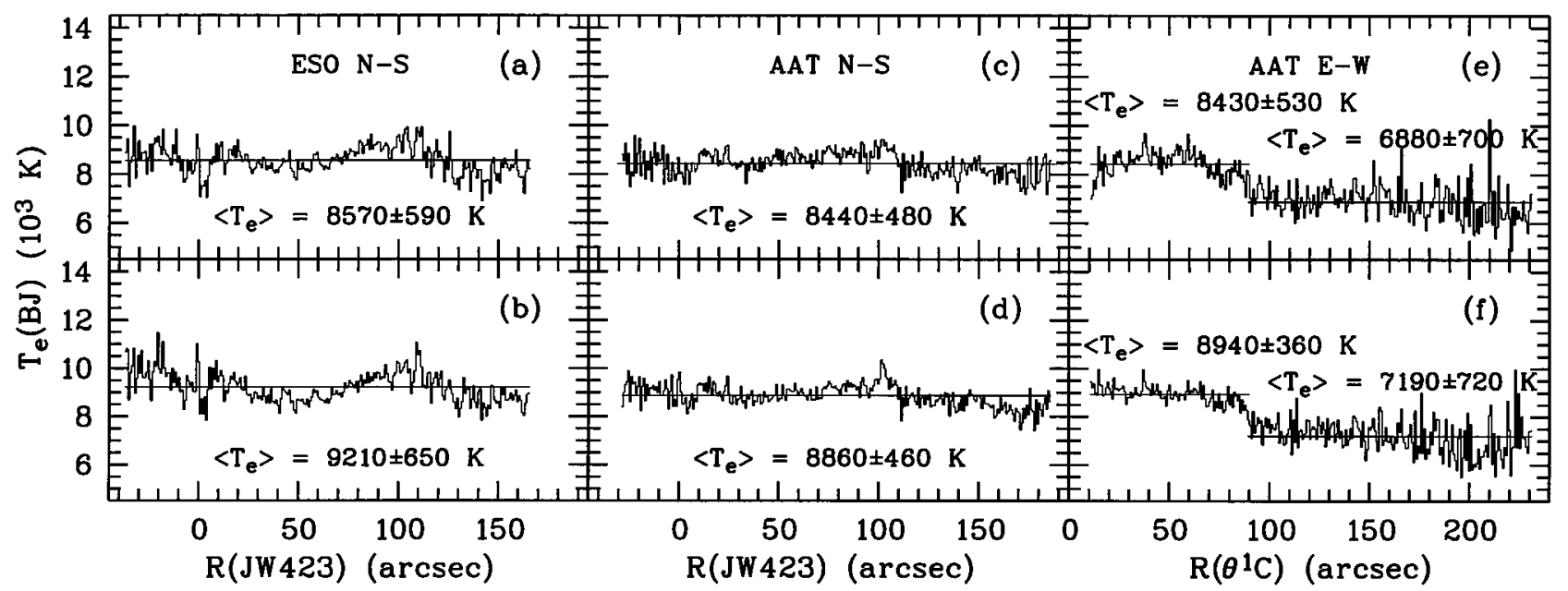

FIG. 5.-Variations of the nebular Balmer discontinuity temperature $T_{e}(\mathrm{BJ})$ in M42 along $(a-d)$ the NS-slit and $(e-f)$ the EW-slit. $(a)$ and $(b)$ were derived from

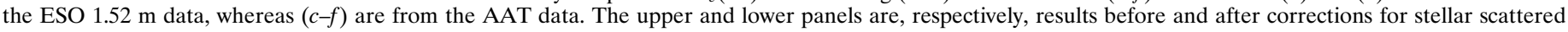

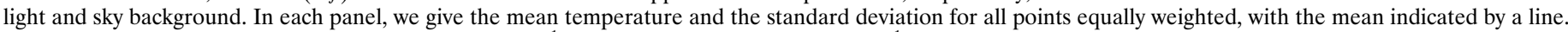
For the EW-slit, two mean values are given, one for $R\left(\theta^{1} \mathrm{C}\right)=10^{\prime \prime}-90^{\prime \prime}$ and another for $R\left(\theta^{1} \mathrm{C}\right)=90^{\prime \prime}-230^{\prime \prime}$. See also the caption to Fig. 1 .

scattered light contribution, the derived temperatures are higher than those without the correction, contrary to previous expectations (Peimbert 1971). For the NS-slit, the results derived from the ESO $1.52 \mathrm{~m}$ data and from the AAT data show excellent agreement. Across the whole 3.5 slit length, $T_{e}(\mathrm{BJ})$ shows only very small variations, of a few hundred $\mathrm{K}$. The average temperature is about $9000 \mathrm{~K}$, in excellent agreement with the value deduced from the integrated spectra (Table 1). The small variations might be real, as they can be seen in both data sets. Along the AAT EW-slit, two regions of slightly different mean temperature can be seen, one of $8900 \mathrm{~K}$ from $10^{\prime \prime}$ to $90^{\prime \prime}$ west of $\theta^{1} \mathrm{C}$ Ori and another of $7200 \mathrm{~K}$ from $90^{\prime \prime}$ west of $\theta^{1} \mathrm{C}$ Ori to the western end of the slit. In parallel to this sudden drop in $T_{e}$, the electron density derived from the [O II] doublet ratio $\lambda 3729 / \lambda 3726$ decreases abruptly from 2600 to $1100 \mathrm{~cm}^{-3}$. A similar change in the [S II] densities was observed previously (B91). Although collisional suppression of the [O III] fine-structure cooling lines can lead to slightly higher $T_{e}$ in the inner, denser regions, photoionization modeling calculations show such an effect is only of the order of a few hundred $\mathrm{K}$. Thus the sudden decrease in $T_{e}$ of $2000 \mathrm{~K}$ is difficult to understand in a picture of M42 as a single H II region ionized by a single central source, i.e., $\theta^{1} \mathrm{C}$ Ori. Figure 15 of $\mathrm{B} 91$ shows that the thickness of the $\mathrm{H}^{+}$zone along the line of sight increases abruptly by a factor of 2 near this position and then increases rapidly beyond. In addition, their Figures 10 and 11 show evidence of irregularities in the ionization structure around this region. One possible explanation is that there is another low surface brightness $\mathrm{H}$ II region, ionized by stars cooler than $\theta^{1} \mathrm{C}$ Ori and thus of lower $T_{e}$, surrounding the "Trapezium" $\mathrm{H}$ II region, and its contribution to the observed fluxes becomes more important beyond $R\left(\theta^{1}\right.$ $\mathrm{C}) \gtrsim 90^{\prime \prime}$. The lowest derived temperatures, of about $5500 \mathrm{~K}$, seen in Figure $5 f$ near $R\left(\theta^{1} \mathrm{C}\right) \sim 200^{\prime \prime}$, correspond to very low surface brightness emission (see Fig. $1 b$ ), so that we do not consider them to be significant.

In conflict with results presented here, WD94 found $T_{e}(\mathrm{BJ})$ to drop from 8400 to $2800 \mathrm{~K}$ between $40^{\prime \prime}$ and $220^{\prime \prime}$ west of $\theta^{1}$ C Ori. As described earlier, by adopting the ratio of the Balmer jump to the intensity of H11, we minimize the effects of many possible systematic errors in determining the temperature, including uncertainties arising from the reddening corrections for M42. As we have observed the four Trapezium stars using the same instrumental setup, we have been able to subtract the scattered light in a consistent way. In contrast, the results of WD94 were more sensitive to reddening corrections. In addition, inspection of their Figure 1 spectrum shows an irregular continuum shape. We conclude that the dramatic variations in $T_{e}(\mathrm{BJ})$ across M42 that they reported are not real.

\section{REFERENCES}

Baldwin, J. A., et al. 1991, ApJ, 374, 580 (B91)

Brown, R. L., \& Mathews, W. G. 1970, ApJ, 160, 939

Cardelli, J. A., Clayton, G. C., \& Mathis, J. S. 1989, ApJ, 345, 245

Costero, R., \& Peimbert, M. 1970, Bol. Obs. Tonantzintla Tacubaya, 5, 229

Jones, B. F., \& Walker, M. F. 1988, AJ, 995, 1755

Liu, X. W., \& Danziger, I. J. 1993, MNRAS, 263, 256

Liu, X. W., Storey, P. J., Barlow, M. J., \& Clegg, R. E. S. 1995, MNRAS, 1995, 272,369
Osterbrock, D. E. 1989, Astrophysics of Gaseous Nebulae and Active Galactic Nuclei (Mill Valley: University Science Books) Peimbert, M. 1967, ApJ, 150, 825

. 1971, Bol. Obs. Tonantzintla Tacubaya, 6, 29

Peimbert, M., Storey, P. J., \& Torres-Peimbert, S. 1993, ApJ, 414, 626

Storey, P. J., \& Hummer, D. G. 1995, MNRAS, 272, 41

Walsh, J. R. 1993, ST-ECF Newsletter, 19, 6

Walter, D. K., \& Dufour, R. J. 1994, ApJ, 434, L29 (WD94) 